\title{
Tetrandrine alleviates cerebral ischemia/reperfusion injury by suppressing NLRP3 inflammasome activation via Sirt-1
}

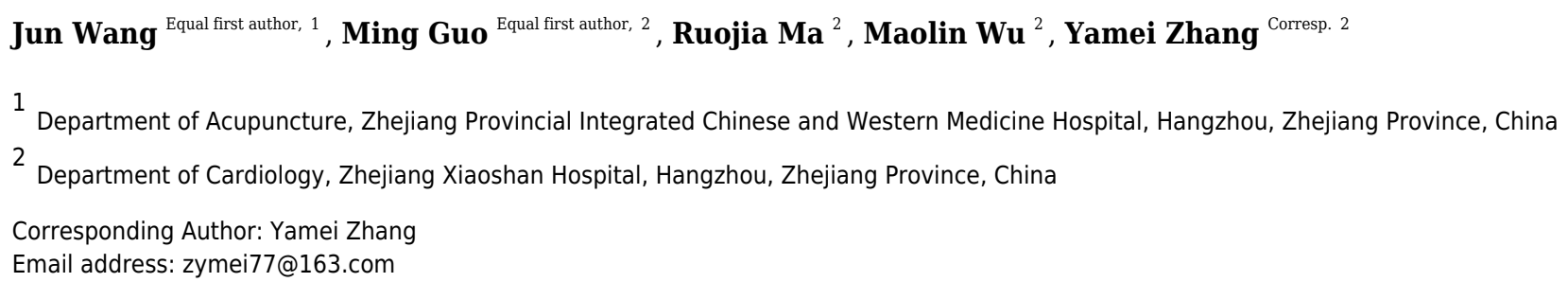

Background \& Aims. Tetrandrine (Tet) has been reported to have anti-inflammatory effects and protect from the ischemic strokes. The NLRP3 inflammasome plays a key role in cerebral ischemia/reperfusion (I/R)-induced inflammatory lesions. However, the molecular mechanisms of Tet related to the progression of cerebral ischemia are still unclear. Therefore, the aim of this study was to investigate the possible effects of Tet on cerebral ischemia and the related mechanisms involved in NLRP3 inflammasome.

Methods. C57BL/6J mice used as a cerebral I/R injury model underwent middle cerebral artery occlusion (MCAO) for $2 \mathrm{~h}$ following reperfusion for $24 \mathrm{~h}$. Tet (30 mg/kg/day, i.p.) was administered for seven days and 30 min before and after MCAO. Their brain tissues were evaluated for NLRP3 inflammasome and Sirtuin-1 (Sirt-1) expression. An intracerebroventricular injection of Sirt-1 siRNA was administered to assess the activation of the NLRP3 inflammasome. Results. Tet significantly reduced the neurological deficits, infarction volume, and cerebral water content in MCAO mice. Moreover, it inhibited I/Rinduced over expression of NLRP3, cleaved caspase-1, interleukin (IL)-1ß, IL-18, and Sirt-1. Sirt-1 knockdown with siRNA greatly blocked the Tet-induced reduction of neurological severity score and infarct volume, and reversed the inhibition of NLRP3 inflammasome activation. Conclusion. Our results demonstrate that Tet has benefits for cerebral I/R injury, which are partially related to the suppression of NLRP3 inflammasome activation via upregulating Sirt-1. 


\section{Tetrandrine alleviates cerebral ischemia/reperfusion injury by}

\section{2 suppressing NLRP3 inflammasome activation via Sirt-1}

3 Wang Jun ${ }^{2, *}$, Guo Ming ${ }^{1, *}$, Ma Ruojia ${ }^{1}$, Wu Maolin ${ }^{1}$, Zhang Yamei ${ }^{1, \#}$

$4{ }^{1}$ Department of Cardiology, Zhejiang Xiaoshan Hospital, Hangzhou, 311200, China

$5{ }^{2}$ Department of Acupuncture, Zhejiang Provincial Integrated Chinese and Western Medicine

6 Hospital, Hangzhou, 310003, China

$7 \quad$ *These authors contributed equally to this work.

8

9 Corresponding Author:

10 Zhang Yamei ${ }^{1, \#}$

11728 Yucai North Road, Hangzhou, Zhejiang Province, 311200, China

12 Email address: zymei77@163.com

13

14

15

16

17

18

19

20

21

22

23

24

25

26

27

28

29

30

31

32 
33 Abstract

34 Background \& Aims. Tetrandrine (Tet) has been reported to have anti-inflammatory effects and 35 protect from the ischemic strokes. The NLRP3 inflammasome plays a key role in cerebral 36 ischemia/reperfusion (I/R)-induced inflammatory lesions. However, the molecular mechanisms 37 of Tet related to the progression of cerebral ischemia are still unclear. Therefore, the aim of this 38 study was to investigate the possible effects of Tet on cerebral ischemia and the related 39 mechanisms involved in NLRP3 inflammasome. Methods. C57BL/6J mice used as a cerebral I/R injury model underwent middle cerebral artery occlusion (MCAO) for $2 \mathrm{~h}$ following reperfusion for $24 \mathrm{~h}$. Tet $(30 \mathrm{mg} / \mathrm{kg} / \mathrm{day}$, i.p.) was administered for seven days and $30 \mathrm{~min}$ before and after MCAO. Their brain tissues were 43 evaluated for NLRP3 inflammasome and Sirtuin-1 (Sirt-1) expression. An intracerebroventricular injection of Sirt-1 siRNA was administered to assess the activation of the 45 NLRP3 inflammasome.

Results. Tet significantly reduced the neurological deficits, infarction volume, and cerebral water content in MCAO mice. Moreover, it inhibited I/R-induced over expression of NLRP3, cleaved caspase-1, interleukin (IL)-1 $\beta$, IL-18, and Sirt-1. Sirt-1 knockdown with siRNA greatly blocked the Tet-induced reduction of neurological severity score and infarct volume, and reversed the inhibition of NLRP3 inflammasome activation.

Conclusion. Our results demonstrate that Tet has benefits for cerebral I/R injury, which are partially related to the suppression of NLRP3 inflammasome activation via upregulating Sirt-1.

53 Subjects Cognitive Disorders, Pharmacology

54 Keywords Cerebral ischemia, Ischemia/reperfusion injury, Tetrandrine, NLRP3 inflammasome, 55 Silent information regulator-1 


\section{Introduction}

66 Ischemic strokes are a leading cause of disability and death worldwide, and place a heavy burden

67 on patients and society (Hou et al., 2019). Although promising researches on the mechanisms of 68 stroke have been conducted in recent years, neuroprotective strategies for clinically application 69 are still lacking. Therefore, seeking effective candidates has become the focus of research. The 70 pathophysiological mechanisms of stroke, especially cerebral ischemia/reperfusion (I/R) injury, 71 are involved in energy metabolism impairment, glutamate/neurotoxin release, autophagy, and 72 inflammation (Guyot et al., 2000). Inappropriate immune response and the inflammatory cascade 73 have been increasingly recognized as important pathological factors influencing I/R injury. The 74 early phase of I/R activates cerebral immune cells, such as microglia, which are responsible for 75 the generation of inflammatory mediators and immunity activation(Schmidt et al., 2016).

76 It is well established that inflammasomes are involved in several brain disorders, such as 77 Alzheimer's disease, Parkinson's disease, epilepsy, and stroke (Aminzadeh et al., 2018; Slowik et 78 al., 2018). The NOD-like receptor pyrin domain-containing 3 (NLRP3), is known as a major component of the inflammasome. It is an intracellular multiprotein signaling complex that includes the NLRP3 scaffold, the adaptor protein PYCARD/ASC, and caspase-1. Once activated, the inflammasome prompts the activation of caspase- 1 and converts pro-interleukin (IL)-1 $\beta$ and pro-IL-18 into their mature forms, which can aggravate inflammatory reactions (Mehto et al., 2019). Various stimuli, such as the release of triphosphate (ATP) by nigericin and injury cells, can trigger the activation of the NLRP3 inflammasome (Chen \& Chen, 2018). NLRP3 and the inflammasome pathways have been shown to be related to inflammation-associated diseases such as atherosclerosis, type II diabetes mellitus and cancer (Karasawa \& Takahashi, 2017; Rovira-Llopis et al., 2018; Wei et al., 2014). Accumulating evidence indicates that the NLRP3 inflammasome plays a decisive role in the development of cerebral I/R injury (Qiu et ai., 2016; Wang et al., 2015). In addition, microglia, innate immune cells in the brain, express NLRP3 to mediate inflammatory cytokine production. The NLRP3 pathway is therefore considered a beneficial target for cerebral I/R injury.

92 Tetrandrine (Tet) is a unique alkaloid extracted from the root of a Chineseherb called Radix 93 Stephania tetrandra. Tet has drawn considerable attention for its anti-tumor (Chen et al., 2009), 94 anti-inflammatory (Li et al., 2018), and analgesic activity (Zhang et al., 2001), and has been 95 widely used for these purposes since ancient times. Studies have shown Tet to be a 
96 neuroprotective agent against ischemic stroke (Sun \& Liu et al., 1995; Ruan et al., 2013). In a 97 middle cerebral artery occlusion (MCAO) mouse model, treatment with tetrandrine was found to 98 reduce infarct volume and brain water content (Ruan et al., 2013). Furthermore, Tet suppresses 99 the production of pro-inflammatory mediators in ischemia in vivo (Chen et al., 2009). However, 100 the possible impact of Tet on the NLRP3 pathway in mice with cerebral ischemia has not yet 101 been reported.

102 Sirtuin-1 (Sirt-1), a member of the sirtuin enzyme family, which includes seven proteins, is 103 distributed in the central nervous system (CNS) of mammals. Several studies have indicated a 104 significant role of Sirt-1 in the neuroprotective mechanism (Kaur et al., 2015; Wang et al., 2019). 105 At the molecular level, it promotes interaction with DNA and several substrates deacetylated and 106 downregulates NLRP3 inflammasome activation in renal epithelial cells (Chou et al., 2019). The 107 role of Sirt-1 expression in regulating the activition of the NLRP3 inflammasome induced by 108 cerebral ischemia remains to be established.

109 In this study, we investigated whether Tet administration has the neuroprotective effects on 110 cerebral I/R injury in an MCAO mouse model. In addition, we sought to reveal the relationship 111 between this neuroprotective effect and Sirt-1-mediated NLRP3 inflammasome activation in 112 vivo. 


\section{Materials \& Methods}

131 Animals. Male C57BL/6J mice (weight $25 \pm 3 \mathrm{~g}$ ) were purchased from Zhejiang Academy of 132 Medical Sciences, Hangzhou, China with experimental animal use license SYXK 2014-0008. All 133 mice were maintained in a $12 \mathrm{~h}$ light-dark cycle, $22-25{ }^{\circ} \mathrm{C}$ and relative humidity $55 \pm 5 \%$ 134 environment, and were free access to water. All experimental animals were performed in strict 135 compliance with the National Institutes of Health Guide for the Care and Use of Laboratory 136 Animals. Procedures were approved by the Institutional Animal Care and Use Committee of the 137 Zhejiang Academy of Medical Sciences. The experimental procedures were approved by the 138 Ethics Committee of Laboratory Animal Care and Welfare, Zhejiang Academy Medical 139 Sciences, with the proved number 2018-143.

140 Experimental protocols. A total of 45 mice were randomly divided into three groups $(\mathrm{n}=6$ for

141 TTC staining; $\mathrm{n}=6$ for behavioral test and protein extraction; $\mathrm{n}=3$ for immunofluorescence 142 analysis): sham operation (sham, $\mathrm{n}=15$ ), MCAO with vehicle (vehicle, $\mathrm{n}=15$ ) or tetrandrine 143 (MCAO + Tet, $\mathrm{n}=15$ ). Tetrandrine (Sigma-Aldrich, St. Louis, MO, USA, PHL89321) was 144 freshly prepared in normal saline. The mice in the MCAO + Tet group received a Tet dose of 30 $145 \mathrm{mg} / \mathrm{kg}$ intraperitoneally (Ruan et al., 2013) once a day for seven days before surgery and $30 \mathrm{~min}$ 146 before and after inducing ischemia. The vehicle group was injected with an equal volume of 147 normal saline. The mice were assigned neurological severity scores $24 \mathrm{~h}$ after MCAO surgery. 148 All mice were then anesthetized with ketamine $(100 \mathrm{mg} / \mathrm{kg}$, i.p. $)$ and sacrificed by cervical 149 dislocation. The brains were harvested to measure cerebral infarct volume and brain water 150 content (Figure 1A). In addition, an intracerebroventricular injection of Sirt-1 small interfering 151 RNA (siRNA) was administered to C57BL/6J mice to inhibit cerebral Sirt-1 expression. Another 15248 mice were randomly divided into two groups: 18 mice for scrambled siRNA injection and 30 153 mice for Sirt-1 siRNA injection. After 48 h, all 48 mice were subjected to MCAO. Furthermore, 154 a Tet group $(n=15)$ and a Tet + Sirt-1 siRNA group $(n=15)$ were treated with Tet as described 155 above, and mice in a Sirt-1 siRNA group $(n=15)$ were received an equal volume of normal 156 saline intraperitoneally. Three mice with scrambled siRNA injection and MCAO were used as 157 controls for Western blot analysis. At the end of the experiment, the surviving animals were 158 sacrificed. 
159 Establishing the cerebral I/R injury induced by $M C A O$. The operating procedure for transient 160 focal cerebral ischemia was previously described by Liu et al (2018). Briefly, the mice were 161 deeply anesthetized, and a midline incision in the neck was made to expose the right external 162 carotid artery (ECA) and the right internal carotid artery (ICA). Silicone-coated nylon 163 monofilament $(0.28 \mathrm{~mm}$ in diameter $)$ was gently inserted from the ECA into the ICA lumen 164 until a 15 - $19 \mathrm{~mm}$ intraluminal thread obstructed the origin of the MCA for $90 \mathrm{~min}$. Then, the 165 nylon was withdrawn to restore blood flow. In the sham group, the mice underwent the same 166 operating procedure without thread insertion.

167 Assignment of neurologic severity score. Twenty-four hours after the ischemic operation, to 168 assess neurological defects, modified neurological severity scores (mNSS) were assignedby a 169 blinded investigator (Chen et al., 2017). The tests evaluated the motion, sensation, reflex, muscle 170 state, abnormal movement, vision, tactile sense and balance systems of the mice. The scores 171 were assigned on a scale from 0 to 18 , where 0represented no evident neurological deficits, and 17218 represented severe deficits.

173 Measurement of brain water content. After the neurological functions were evaluated, the mice 174 were euthanized, and their brains were removed immediately. The cerebral cortex (at $2 \mathrm{~mm}$ 175 around the craniotomy) was isolated, and blood and cerebrospinal fluid were removed with filter 176 paper. After the wet weight was measured using an analytical balance, the samples were dried in 177 an oven at $100{ }^{\circ} \mathrm{C}$ for $24 \mathrm{~h}$. The dry weight was then measured with an analytical balance. The 178 brain water content was calculated according to as the formula $\%=$ (wet weight - dry 179 weight)/wet weight $\times 100$.

180 Measurement of cerebral infarct volume. The cerebral infarct volume was determined using 181 2,3,5-triphenyltetrazolium chloride (TTC) staining as previously described (Liu et al., 2018). The 182 brains were cut into five coronal sections, which were incubated in $2 \% \mathrm{TTC}$ at $37^{\circ} \mathrm{C}$ for $30 \mathrm{~min}$. 183 All slides were then fixed in $4 \%$ paraformaldehyde buffer for $24 \mathrm{~h}$. The infarct and total 184 hemispheric areas were measured using the ImageJ analysis software (National Institutes of 185 Health, Bethesda, MD, USA). The ischemic volume was calculated as the percentage of cerebral 186 ischemic volume to the total volume of the sections.

187 Immunofluorescence analysis. Ischemic hippocampal tissue was fixed in formaldehyde before 188 being embedded in paraffin and cut into $4 \mu \mathrm{m}$ sections. The samples then underwent 189 deparaffinization with dimethyl benzene, gradient alcohol dehydration, and antigen retrieval 
190 according to the citric acid buffer/microwave protocol. The sections were incubated overnight 191 with primary antibodies for Iba-1 (diluted 1:200; Abcam, Cambridge, UK) and NLRP3 (diluted 192 1:200; Invitrogen, Grand Island, NY, USA) at $4{ }^{\circ} \mathrm{C}$. The slides were then washed with 193 phosphate-buffered saline (PBS) and incubated with goat anti-rabbit IgG antibody (diluted 194 1:200; Invitrogen, Grand Island, NY, USA) at room temperature for $1 \mathrm{~h}$. Following staining with 195 4',6-diamidino-2-phenylindole (DAPI; diluted 1:300; Molecular Probes/Invitrogen Life 196 Technologies, Eugene, OR, USA), the slides were examined with a fluorescence microscope 197 (Leica Microsystems, Wetzlar, Germany). Image analysis was performed using Image J 198 (National Institutes of Health, Bethesda, MD, USA). The density of Iba-1-positive cells and cels1 199 with Iba-1 localization of NLRP3 was measured (cells $/ \mathrm{mm}^{2}$ ). One section per mice and three 200 mice per group were evaluated.

201 determination of inflammatory cytokines. Inflammatory factors in cerebral tissue were 202 determined with enzyme-linked immunosorbent assay (ELISA) kits (Anogen, Mississauga, 203 Ontario, Canada) according to the manufacturer's instructions. Briefly, IL-1 $\beta$ and IL-18 in both 204 standards and samples were performed with monoclonal anti-mouse IL-1 $\beta$ and IL-18 as primary 205 antibodies. All OD values were converted into corresponding concentration values.

206 Intracerebroventricular injection of Sirt-1 siRNA. Sirt-1 siRNA (sense primer, 5'207 GCAGAUUAGUAAGCGUCUUTT-3'; $\quad$ antisense 208 AAGACGCUUACUAAUCUGCTT-3') was designed and synthesized by GenePharma 209 Corporation (Shanghai, China). A scrambled siRNA (sense primer, 5'210 GCGCCAGUGGUACUUAAUATT-3'; 211 UAUUAAGUACCACUGGCGCTT-3') was synthesized without a target sequence for the 212 control. An intracerebroventricular injection of siRNA was administered according to the method 213 described by Wang et al., (2019). The mice were deeply anesthetized. Hair was removed from 214 the center of the head after swabbing with povidone iodine and 75\% ethanol. The mice were then 215 placed on a stereotaxic apparatus. A $25 \mu \mathrm{L}$ Hamilton syringe was fixed on the stereotaxic 216 apparatus and inserted perpendicularly at $1.0 \mathrm{~mm}$ posterior to the bregma and $2.0 \mathrm{~mm}$ lateral to 217 the midline and to a depth of $3.5 \mathrm{~mm}$ beneath the surface of the skull. Next, $10 \mu \mathrm{L}$ of Sirt-1 218 siRNA diluent $(2 \mu \mathrm{g} / \mu \mathrm{L})$ was injected into the right lateral ventricle at a rate of $1 \mu \mathrm{L} / \mathrm{min}$. Upon 219 completion, the needle was gently withdrawn. Forty-eight hours later, the mice were subjected to 220 MCAO. 
221 Western blot analysis. Protein was extracted from brain tissue with radioimmunoprecipitation 222 assay lysis buffer (Dallas, TX, USA), and a total of $30 \mu \mathrm{g}$ of protein was separated by sodium 223 dodecyl sulfate-polyacrylamide gel electrophoresis. The protein was then transferred to a 224 nitrocellulose membrane and blocked in 10\% skimmed milk at room temperature for $1 \mathrm{~h}$. The 225 membranes were incubated overnight with primary antibodies against NLRP3 (1:500; 226 Invitrogen, Grand Island, NY, USA), cleaved caspase-1 (1:500, Abcam, Cambridge, UK), Sirt-1 227 (1:300, Cell Signaling Technology, Danvers, MA, USA), and glyceraldehyde 3-phosphate 228 dehydrogenase (GAPDH; 1:5,000; Bioworld Technology, St Louis Park, MN, USA) at $4{ }^{\circ} \mathrm{C}$. The 229 membranes were then washed with PBS and incubated with horseradish peroxidase-conjugated $230 \operatorname{IgG}$ (1:5,000, Cell Signaling Technology, Danvers, MA, USA) secondary antibodies at room 231 temperature for $1 \mathrm{~h}$. The protein bands were visualized using an ECL Western Blotting Detection 232 System (Millipore, Billerica, MA, USA) and normalized for GAPDH expression.

233 Statistical analysis. The results were expressed as means \pm standard error of the mean (SEM). 234 Statistical analysis was performed by one-way analysis of variance (ANOVA) followed by 235 Newman-Keuls multiple comparison tests. A value of $P$ less then 0.05 was considered 236 statistically significant.

237

238

239

240

241

242

243

244

245

246

247

248

249

250

251

252

253 


\section{Results}

257 Tet ameliorated cerebral I/R injury. To explore the neuroprotective effects of Tet against 258 cerebral I/R injury, cerebral infarct volume, mNSS scores, and brain water content were 259 examined. As shown in Figure $1 \mathrm{~B}$ and $\mathrm{C}$, we observed that infarct volume in the MCAO + Tet

260

261

262

263

264

265

266

267

268

269

270

271

272

273

274

275

276

277

278

279

280

281

282

283

284

group was clearly smaller than in the vehicle group. Similarly, the Tet treatment significantly improved the neurological severity scores and decreased the brain water content in the MCAO + Tet group compared with the vehicle group (Figure 1D and E). Taken together, these data indicated that the Tet treatment had protective effects on cerebral I/R injury.

Tet inhibited the activation of the NLRP3 inflammasome in the hippocampal microglia of ischemic brains. To investigate the influence of Tet on NLRP3 inflammasome in hippocampal microglia, immunofluorescence staining was used. The results showed that the density of Iba-1positive cells in the hippocampus region, corresponding to the number of microglia, was higher in the vehicle group than in the sham group (Figure 2B, F, and M). Stronger and more extensive staining for NLRP3 appeared in the hippocampus region of mice in the vehicle group compared with those in the sham group (Figure $2 \mathrm{~A}$ and $\mathrm{E}$ ), and their microglia were activated, showing a dramatic increase in positive staining for NLRP3 (Figure 2D, H, and N). However, the Tet treatment greatly reduced the number of Iba-1-positive cells and the percentage of cells with Iba1 localization with NLRP3 in the MCAO + Tet group compared with the vehicle group (Figure 2E-N).

Tet inhibited NLRP3 -derived inflammation and upregulated Sirt-1 expression in cerebral I/R injury. To clarify the protective effects of Tet related to NLRP3 -derived inflammation, we used Western blot to detect the protein levels of NLRP3 and cleaved caspase-1, and ELISA to detect IL-1 $\beta$ and IL-18 levels in brain tissue. The data showed that NLRP3, cleaved caspase-1, IL-1 $\beta$, and IL-18 levels were significantly elevated in the vehicle group compared with the sham group. Importantly, these increases were dramatically inhibited in the MCAO + Tet group compared to the vehicle group (Figure 3A-E). To determine the effect of Tet on Sirt-1 expression in response to cerebral I/R injury, we evaluated the protein level of Sirt-1. The protein levels of Sirt-1 were significantly lower in the cerebral I/R-induced group than in the sham group. Treatment with Tet upregulated the expression of Sirt-1 in the MCAO + Tet group compared to the vehicle group 
285 (Figure $3 \mathrm{~F}$ and $\mathrm{G}$ ). These results suggested that $\mathrm{I} / \mathrm{R}$-induced cerebral injury improvement 286 through Tet was related to the inhibition of NLRP3-regulated release of inflammatory cytokines 287 and the upregulation of Sirt-1.

288 Tet suppressed NLRP3 inflammasome activation through Sirt-1.

289 To elucidate the molecular mechanisms of Sirt-1 on the activation of NLRP3 inflammasome 290 components in response to cerebral I/R injury, cerebral Sirt-1 was partially knocked down by 291 siRNA. Compared with the Tet group, Sirt-1 siRNA pretreatment increased cerebral infarct 292 volume and neurological severity score. There were no significant differences in infarct volume 293 and neurological score between the Sirt-1 siRNA group and the Tet + Sirt-1 siRNA group 294 (Figure 4A-C). In addition, Sirt-1 siRNA significantly reduced the protein level of Sirt-1 in the 295 mice that underwent MCAO (Figure 4D-E). These results indicated that Sirt-1 played a role in 296 Tet-mediated neuroprotective effects under I/R stimulation. Furthermore, we found that Sirt-1 297 siRNA markedly increased the number of Iba-1-positive cells and the percentage of NLRP3 in 298 hippocampal microglia in the Sirt-1 siRNA group compared to the Tet group (Figure 5A-E). 299 Similarly, Sirt-1 siRNA effectively upregulated IL-1 $\beta$ and IL-18 in brain tissue compared to the 300 Tet group (Figure 5F and G). Moreover, Tet-induced suppression of NLRP3-derived 301 inflammation, including NLRP3, IL-1 $\beta$, and IL-18, was significantly abolished by partial Sirt-1 302 knockdown (Figure 5A-G). Overall, these findings suggested that Tet downregulated cerebral 303 I/R-induced NLRP3 inflammasome expression through upregulating Sirt-1.

304

305

306

307

308

309

310

311

312

313

314

315

316 


\section{Discussion}

320 In the present study, we demonstrated that treatment with tetrandrine in mice exerted

321 neuroprotective effects following MCAO. First, it significantly lowered the neurological severity 322 scores and reduced infarct volume and brain edema in the MCAO + Tet group compared with the 323 vehicle group. Second, Tet inhibited NLRP3 inflammasome activation, as evidenced by 324 suppressed expression of NLRP3 in microglial cells and the reduced levels of NLRP3, cleaved 325 caspase-1, IL-1 $\beta$, and IL-18 in cerebral tissue. Finally, Tet reduced NLRP3-derived 326 inflammation via upregulating Sirt-1, indicating that the protective effects of Tet on cerebral I/R injury in mice were related to Sirt-1 and NLRP3.

Ischemic stroke occurs due to distinctly reduced blood flow to the brain, accompanied by activating the ischemic cascade, which causes to serious neuronal injury. Blood reperfusion is thought to cause more severe secondary tissue damage which induces an inflammatory response

331 332 333 to the latter process, leading to additional injury to adjacent brain tissue (Mizuma \& Yenari et al., 2017). Unfortunately, few therapy options are available for minimizing tissue damage after a stroke. thus, It is therefore urgent to explore effective agents.

Tet, a natural bisbenzylisoquinoline alkaloid compound, exhibits significant bioavailability and has anti-cancer (Chen et al., 2009), anti-inflammatory (Li et al., 2018), and cytoprotective effects (Zhang et al., 2017). Such pharmacological effects have clinical applications for several conditions, including arrhythmia, silicosis, inflammation, and occlusive cardiovascular disorders (Chen et al., 2011). Furthermore, Tet has been reported to attenuate heart (Zhang et al., 2017), liver (Liu et al., 2004), and small bowl (Chen et al., 2009) from I/R injury. Zhang et al., reported that Tet had beneficial effects on I/R-induced injury in cardiac cell models and the mechanisms involved in the JAK3/STAT3/HK II signaling pathway (Zhang et al., 2017). However, the effects of Tet on ischemic stroke have yet to be determined. Tet has the advantages of being extremely fat-soluble and hydrophobic and having a low molecular weight, which allow it to cross the blood brain barrier (Chen et al., 2011). In MCAO mice, Tet (30 mg/kg) contributed to an improvement of cerebral I/R injury partially through regulating GRP78, DJ-1, and HYOU1 protein expression (Ruan et al., 2013). In a global cerebral I/R gerbil model, pretreatment with Tet alleviated cortex and hippocampus structural abnormalities (Sun \& Liu et al., 1995). In this 
348 study, we used C57BL/6 mice subjected to MCAO as an experimental model of focal cerebral $349 \mathrm{I} / \mathrm{R}$ injury. We showed that Tet treatment $(30 \mathrm{mg} / \mathrm{kg} /$ day $)$ for seven consecutive day reduced 350 neurological severity scores, infarct volume, and brain water content. Nevertheless, the 351 mechanisms that underlie ischemic stroke are still not fully understood.

352 Numerous studies have shown that inflammation is closely associated with the development of 353 cerebral I/R injury (Meng et al., 2019; Mizuma \& Yenari et al., 2017). NLRP3 inflammasome 354 activation plays a critical pathogenic role in strokes (Qiu et al., 2016; Wang et al., 2015). The 355 expression of NLRP3 is regarded as a rate-limiting element for inflammasome activation. 356 Generally, the NLRP3 inflammasome is largely located in microglia in the CNS. Previous 357 studies have demonstrated that ischemic stroke can induce microglia activation and promote 358 NLRP3 expression, resulting in neuronal cell death (Xu et al., 2018; Wang et al., 2017). The 359 activated microglia subsequently release chemokines, cytotoxic mediators, and cytokines, 360 including IL-1 $\beta$, TNF- $\alpha$ and IL-6, triggering the inflammatory cascade after an ischemic stroke, 361 thus further exacerbating neuroinflammatory damage. It has been shown that downregulation of 362 NLRP3 inflammasome has a neuroprotective effect against ischemic strokes (Wang et al., 2019). 363 In addition, NLRP3 knockdown by siRNA effectively ameliorated cerebral ischemia damage (He 364 et al., 2017). In line with these studies, we found that the percentage of cells with Iba-1 365 localization of NLRP3 were significantly enhanced in the hippocampus; correspondingly, the 366 levels of NLRP3, cleaved caspase-1, IL-1 $\beta$, and IL-18 were elevated in the MCAO mice 367 compared with the control group. Above all, these findings suggest that suppressing NLRP3 368 inflammasome activation might be a beneficial target for ischemic insults.

369 Tet exhibits anti-inflammatory properties in peripheral reflected by inhibiting $\mathrm{T}$ cells, B cells, 370 and the production of cytokines and inflammatory mediators (Chen et al., 2009; Li et al., 2003). 371 Furthermore, it has been shown to suppress overexpression of ICAM-1, TNF- $\alpha$, IL-1 $\beta$, and IL-6 372 in an acute pancreatitis rat model and a transplanted small bowel pig model (Chen et al., 2009; 373 Wang et al., 2004). It would thus be worth exploiting the anti-inflammatory activity of Tet after 374 an ischemic stroke. In this study, treatment with Tet drastically suppressed the activation of 375 NLRP3 inflammasome in the MCAO group.

376 To understand how Tet inhibits the NLRP3 inflammasome, we focused on protein deacetylase 377 Sirt-1 because of its high expression in the CNS. Importantly, Sirt-1 plays a critical role in 378 neuroprotective mechanisms against cerebral ischemic injury and exhibits anti-inflammatory 
379 activity through mediating NLRP3 inflammasome activation (Yang et al., 2015; Ma et al., 2015).

380 In our study, Tet treatment remarkably increased Sirt-1 expression in MCAO mice. Intriguingly, 381 Sirt-1 knockdown using siRNA significantly reduced the neuroprotective effects and reversed 382 the suppression of NLRP3 inflammasome activation mediated by Tet. Our results indicate that 383 Tet exerts neuroprotective effects against ischemic stroke injury partly through inhibiting the 384 activation of the NLRP3 inflammasome via upregulating Sirt-1.

\section{Conclusions}

386 Our results showed that treatment with Tet can protect against cerebral I/R-induced brain tissue 387 injury in mice, which is possibly associated with the suppression of Sirt-1-mediated NLRP3 388 inflammasome activation. Our study demonstrates the potential of Tet for ameliorating cerebral 389 I/R injury, suggesting its clinical advantages for cardiovascular disease therapy. Nevertheless, 390 further investigations are needed to understand more precisely the mechanism underlying Tet 391 with neuroinflammatory and its possible applications in ischemic stroke therapy.

392

393

394

395

396

397

398

399

400

401

402

403

404

405

406

407

408

409

410

411 
412

413

414 References

415 Aminzadeh M, Roghani M, Sarfallah A, Riazi GH. 2018. TRPM2 dependence of ROS416 induced NLRP3 activation in Alzheimer's disease. Int. Immunopharmacol. 54: 78-85 DOI 417 10.1016/j.intimp.2017.10.024.

418 Chen J, Chen ZJ. 2018. Ptdlns4P on dispersed trans-golginetwork mediates NLRP3 419 inflammasome activation. Nature. 564(7734): 71-76 DOI 10.1038/s41586-018-0761-3.

420 Chen X, Wu S, Chen C, Xie B, Fang Z, Hu W, Chen J, Fu H, He H. 2017. Omega-3 421 polyunsaturated fatty acid supplementation attenuates microglialinduced inflammation by

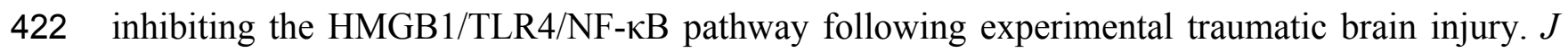
423 Neuroinflammation. 14(1):143 DOI 10.1186/s12974-017-0917-3.

424 Chen Y, Chen JC, Tseng SH. 2009. Effects of tetrandrine plus radiation on neuroblastoma 425 cells. Anticancer Res. 29(8):3163-71.

426 Chen Y, Chen JC, Tseng SH. 2009. Tetrandrine suppresses tumor growth and angiogenesis of 427 gliomas in rats. Int J Cancer. 124(10):2260-9 DOI 10.1002/ijc.24208.

428 Chen Y, Tsai YH, Tseng SH. 2011. The potential of tetrandrine as a protective agent for 429 ischemic stroke. Molecules. 16(9):8020-32 DOI 10.3390/molecules16098020.

430 Chen Y, Wu JM, Lin TY, Wu CC, Chiu KM, Chang BF, Tseng SH, Chu SH. 2009. 431 Tetrandrine ameliorated reperfusion injury of small bowel transplantation. J. Pediatr. Surg. 432 44(11): 2145-52 DOI 10.1016/j.jpedsurg.2009.06.002.

433 Chou X, Ding F, Zhang X, Ding X, Gao H, Wu Q. 2019. Sirtuin-1 ameliorates cadmium434 induced endoplasmic reticulum stress and pyroptosis through XBP-1s deacetylation in human 435 renal tubular epithelial cells. Arch Toxicol. 93(4): 965-986 DOI 10.1007/s00204-019-02415-8.

436 Guyot LL, Diaz FG, O'Regan MH, Ren J, Phillis JW. 2000. The effect of intravenous insulin 437 on accumulation of excitotoxic and other amino acids in the ischemic rat cerebral cortex. 438 Neurosci Lett. 288(1):61-5 DOI 10.1016/s0304-3940(00)01168-x.

439 He Q, Li Z, Wang Y, Hou Y, Li L, Zhao J. 2017. Resveratrol alleviates cerebral 440 ischemia/reperfusion injury in rats by inhibiting NLRP3 inflammasome activation through Sirt1441 dependent autophagy induction. Int Immunopharmacol. 50:208-215 DOI 442 10.1016/j.intimp.2017.06.029. 
443 Hou S, Zhang H, Li B, Luo Y, Han Z, Shang S. 2019. Validation and Evaluation of the Core 444 Nursing Outcomes Evaluation System for Inpatients with Stroke. Int J Nurs Knowl DOI $44510.1111 / 2047-3095.12259$.

446 Karasawa T, Takahashi M. 2017. The crystal-induced activation of NLRP3 inflammasomes in 447 atherosclerosis. Inflamm Regen. 37: 18 DOI 10.1186/s41232-017-0050-9.

448 Kaur H, Kumar A, Jaggi AS, Singh N. 2015. Pharmacologic investigations on the role of Sirt4491 in neuroprotective mechanism of postconditioning in mice. J Surg Res. 197(1):191-200 DOI $450 \quad 10.1016 /$ j.jss.2015.03.010.

451 Li X, Wu Z, He B, Zhong W. 2018. Tetrandrine alleviates symptoms of rheumatoid arthritis in 452 rats by regulating the expression of cyclooxygenase-2 and inflammatory factors. Exp Ther Med. 453 16(3):2670-2676 DOI 10.3892/etm.2018.6498.

454 Li YY, Li XL, Yang CX, Zhong H, Yao H, Zhu L. 2003. Effects of tetrandrine and QYT on 455 ICAM-1 and SOD gene expression in pancreas and liver of rats with acute pancreatitis. World $J$ 456 Gastroenterol. 9(1):155-9 DOI 10.3748/wjg.v9.i1.155.

457 Liu Y, Yang H, Jia G, Li L, Chen H, Bi J, Wang C. 2018. The Synergistic Neuroprotective 458 Effects of Combined Rosuvastatin and Resveratrol Pretreatment against Cerebral 459 Ischemia/Reperfusion Injury. $J$ Stroke Cerebrovasc Dis. 27(6):1697-1704 DOI $460 \quad 10.1016 /$ j.jstrokecerebrovasdis.2018.01.033.

461 Liu Z, Xu Z, Shen W, Li Y, Zhang J, Ye X. 2004. Effect of pharmacologic preconditioning 462 with tetrandrine on subsequent ischemia/reperfusion injury in rat liver. World J Surg. 28(6):620463 4. DOI 10.1007/s00268-004-7172-3.

464 Ma CH, Kang LL, Ren HM, Zhang DM, Kong LD. 2015. Simiao pill ameliorates renal 465 glomerular injury via increasing Sirtlexpression and suppressing NF-kappaB/NLRP3 466 inflammasome activation in high fructose-fed rats. $J$ Ethnopharmacol. 172:108-17 DOI 467 10.1016/j.jep.2015.06.015.

468 Mehto S, Chauhan S, Jena KK, Chauhan NR, Nath P, Sahu R, Dhar K, Das SK, Chauhan 469 S. 2019. IRGM restrains NLRP3 inflammasome activation by mediating its SQSTM1/p62470 dependent selective autophagy. Autophagy. 15(9):1645-1647. DOI $47110.1080 / 15548627.2019 .1628544$. 
472 Meng C, Zhang J, Zhang L, Wang Y, Li Z, Zhao J. 2019. Effects of NLRP6 in Cerebral 473 Ischemia/Reperfusion (I/R) Injury in Rats. J Mol Neurosci. 69(3):411-418 DOI 10.1007/s12031474 019-01370-4.

475 Mizuma A, Yenari MA. 2017. Anti-Inflammatory Targets for the Treatment of Reperfusion 476 Injury in Stroke. Front Neurol. 8:467 DOI 10.3389/fneur.2017.00467.

477 Qiu J, Wang M, Zhang J, Cai Q, Lu D, Li Y, Dong Y, Zhao T, Chen H. 2016. The 478 neuroprotection of Sinomenine against ischemic stroke in mice by suppressing NLRP3 479 inflammasome via AMPK signaling. Int. Immunopharmacol. 40: $492-500$ DOI $480 \quad 10.1016 /$ j.intimp.2016.09.024.

481 Rovira-Llopis S, Apostolova N, Bañuls C, Muntané J, Rocha M, Victor VM. 2018. 482 Mitochondria, the NLRP3 inflammasome, and sirtuins in type 2 diabetes: new therapeutic 483 targets, Antioxid. Redox Signal. 29 (8): 749-791 DOI 10.1089/ars.2017.7313.

484 Ruan L, Huang HS, Jin WX, Chen HM, Li XJ, Gong QJ. 2013. Tetrandrine Attenuated 485 Cerebral Ischemia/Reperfusion Injury and Induced Differential Proteomic Changes in a MCAO 486 Mice Model Using 2-D DIGE. Neurochem Res. 38(9):1871-9 DOI 10.1007/s11064-013-1093-1.

487 Schmidt C, Frahm C, Schneble N, Müller JP, Brodhun M, Franco I, Witte OW, Hirsch E, 488 Wetzker R, Bauer R. 2016. Phosphoinositide 3-Kinase $\gamma$ Restrains Neurotoxic Effects of 489 Microglia After Focal Brain Ischemia. Mol Neurobiol. 53(8):5468-79 DOI 10.1007/s12035-015490 9472-z.

491 Slowik A, Lammerding L, Hoffmann S, Beyer C. 2018. Brain inflammasomes in stroke and 492 depressive disorders: Regulation by oestrogen. $J$ Neuroendocrinol. 30(2) DOI 493 10.1111/jne.12482.

494 Sun F, Liu TP. 1995. Tetrandrine vs nicardipine in cerebral ischemia-reperfusion damages in 495 Gerbils. Zhongguo Yao Li Xue Bao. 16(2):145-8.

496 Wang D, Zhang J, Jiang W, Cao Z, Zhao F, Cai T, Aschner M, Luo W. 2017. The role of 497 NLRP3-CASP1 in inflammasome-mediated neuroinflammation and autophagy dysfunction in 498 manganese-induced, hippocampal-dependent impairment of learning and memory ability. 499 Autophagy. 13(5):914-927 DOI 10.1080/15548627.2017.1293766.

500 Wang G, Lemos JR, Iadecola C. 2004. Herbal alkaloid tetrandrine: From an ion channel 501 blocker to inhibitor of tumor proliferation. Trends Pharmacol Sci. 25(3):120-3 DOI 502 10.1016/j.tips.2004.01.009. 
503 Wang P, Lu Y, Han D, Wang P, Ren L, Bi J, Liang J. 2019. Neuroprotection by nicotinamide

504

505

506

507

508

509

510

511

512

513

514

515

516

517

518

519

520

521

522

523

524

525

526

527

528

529

530

531

532

533 mononucleotide adenylyltransferase 1 with involvement of autophagy in an aged rat model of transient cerebral ischemia and reperfusion. Brain Res. 1723:146391 DOI 10.1016/j.brainres.2019.146391.

Wang X, Li R, Wang X, Fu Q, Ma S. 2015. Umbelliferone ameliorates cerebral ischemiareperfusion injury via upregulating the PPAR gamma expression and suppressing TXNIP/NLRP3 inflammasome. Neurosci. Lett. $\quad 600: \quad 182-187 \quad$ DOI: 10.1016/j.neulet.2015.06.016.

Wang Y, Menga C, Zhang J, Wu J, Zhao J. 2019. Inhibition of GSK-3 $\beta$ alleviates cerebral ischemia/reperfusion injury in rats by suppressing NLRP3 inflammasome activation through autophagy. International Immunopharmacology. $\quad 68 \quad \mathbf{( 2 0 1 9 )} \quad: \quad 234-241$ DOI org/10.1016/j.intimp.2018.12.042.

Wei Q, Mu K, Li T, Zhang Y, Yang Z, Jia X, Zhao W, Huai W, Guo P, Han L. 2014. Deregulation of the NLRP3 inflammasome in hepatic parenchymal cells during liver cancer progression, Lab. Investig. 94 (1): 52-62 DOI 10.1038/labinvest.2013.126.

Xu X, Zhang L, Ye X, Hao Q, Zhang T, Cui G, Yu M. 2018. Nrf2/ARE pathway inhibits ROS-induced NLRP3 inflammasome activation in BV2 cells after cerebral ischemia reperfusion. Inflamm Res. 67(1):57-65 DOI 10.1007/s00011-017-1095-6.

Yang Y, Jiang S, Dong Y, Fan C, Zhao L, Yang X, Li J, Di S, Yue L, Liang G, Reiter RJ, Qu Y. 2015. Melatonin prevents cell death and mitochondrial dysfunction via a SIRT1dependent mechanism during ischemic-stroke in mice. J Pineal Res. 58(1):61-70 DOI 10.1111/jpi.12193.

Zhang TJ, Guo RX, Li X, Wang YW, Li YJ. 2017. Tetrandrine cardioprotection in ischemiareperfusion (I/R) injury via JAK3/STAT3/Hexokinase II. Eur J Pharmacol. 813:153-160 DOI 10.1016/j.ejphar.2017.08.019.

Zhang YH, Fang LH. 2001. Antagonism of morphine-induced antinociception by tetrandrine is dependent on serotonergic mechanisms. Life Sci. 69(12):1429-39.

Figure 1. Tetrandrine (Tet) alleviated middle cerebral artery occlusion (MCAO)-induced injury in mice. (A) Illustration of experimental schedule. The C57BL/6J mice received Tet (30mg/kg, i.p.) or vehicle for 7 days. The mice were then subjected to MCAO, followed by Tet treatment 30 
534 min before and after surgery. The neurobehavioral outcomes and infarct volumes were assessed

535 on day 9. The representative images of TTC-stained brain sections (B) and quantification the 536 infarct area (C) were showed. Neurological scores (D) and brain water content (E) were 537 measured after cerebral ischemia. Values are mean $\pm \mathrm{SEM}$, ( $\mathrm{n}=6$ per group). ${ }^{*} P<0.05$ vs. Sham; $538{ }^{\#} P<0.05$ vs. vehicle.

539

540 Figure 2. Tetrandrine inhibited the activation of the NLRP3 inflammasome in hippocampal 541 microglia of ischemic brain. Brain sections were stained with DAPI (blue), as well as Iba-1 542 (green) or NLRP3 (red) to monitor NLRP3 accumulation. (A-L) Representative 543 immunofluorescent staining in the hippocampus of mice. Scale bar, $100 \mu \mathrm{m}$. Iba-1 positive cells 544 in the hippocampus (M) and percentage of cells with Iba-1 localization of NLRP3 (N) were 545 quantified. Tet, Tetrandrine.

546

547 Figure 3. Tetrandrine inhibited NLRP3 -derived inflammation and upregulated Sirt-1 expression 548 in ischemic brain in mice. (A) Protein levels of NLRP3 and cleaved caspase-1 in brain tissues 549 were measured by western blots and were normalized to GAPDH. The bar graphs show that Tet 550 treatment clearly increased the expression of NLRP3 (B) and cleaved caspase-1 (C). N=3 per 551 group. The levels of IL-1 $\beta$ (D) and IL-18 (E) were analyzed by ELISA. N=6 per group. The 552 representative bands of western blots (F) and quantification the Sirt-1 expression (G) were 553 shown. Treatment with Tet before and after MCAO significantly increased Sirt-1 expression in 554 brain tissues. $\mathrm{N}=3$ per group. Values are mean $\pm \mathrm{SEM},{ }^{*} P<0.05$ vs. Sham; ${ }^{\#} P<0.05$ vs. vehicle. 555 Tet, Tetrandrine.

556

557 Figure 4. The effect of Sirt-1 siRNA pretreatment on infarct volumes and neurobehavioral 558 outcomes in cerebral ischemia in mice. An intracerebroventricular injection of Sirt-1 siRNA was 559 administered to mice to inhibit cerebral Sirt-1 expression, and a scrambled siRNA was injected 560 as a control. (A, B) TTC-stained brain sections showed no significantly different atrophy 561 between Sirt-1 siRNA group and Tet + Sirt-1 siRNA group. $N=6$ per group, ${ }^{*} P<0.05$ vs. Tet. (C) 562 Neurological severity scores were detected after cerebral ischemia. $\mathrm{N}=6$ per group. ${ }^{*} P<0.05$ vs.

563 Tet. (D) Representative western blots showed the protein level of Sirt-1 in mice and was 564 normalized to GAPDH. Sirt-1 siRNA pretreatment significantly inhibited protein level of Sirt-1 
565 in brain tissues. Quantification of the expression level is shown in (E). $\mathrm{N}=3$ per group. ${ }^{*} P<0.05$.

566 Values are mean \pm SEM. Tet, Tetrandrine. NS, no significance.

567

568 Figure 5. Sirt-1 siRNA obviously reverses the effects of Tetrandrine on expression of NLRP3, 569 IL-1 $\beta$ and IL-18 in mice. Tet and Sirt-1 siRNA pretreatment blocked the Tet-induced decrease 570 NLRP3 expression in Iba-1-positive cells in the hippocampus (A-C). Scale bar, $100 \mu \mathrm{m}$. Iba-1 571 positive cells in the hippocampus (D) and percentage of cells with Iba-1 localization of NLRP3 572 (E) were quantified. Sirt-1 siRNA also blocked the Tet-induced reduction of IL-1 $\beta$ (F) and IL-18 573 (G) levels. Values are mean $\pm \mathrm{SEM}$, ( $\mathrm{n}=6$ per group). ${ }^{*} P<0.05$ vs. Tet. Tet, Tetrandrine. NS, no 574 significance.

575 


\section{Figure 1}

Tetrandrine (Tet) alleviated middle cerebral artery occlusion (MCAO)-induced injury in mice.

(A) Illustration of experimental schedule. The C57BL/6J mice received Tet (30mg/kg, i.p.) or vehicle for 7 days. Then, the mice were subjected to MCAO, followed by Tet treatment $30 \mathrm{~min}$ before and after surgery. The neurobehavioral outcomes and infarct volumes were assessed on day 9. The representative images of TTC-stained brain sections (B) and quantification the infarct area (C) were shown. Neurological scores (D) and brain water content (E) were measured after cerebral ischemia. Values are mean $\pm \mathrm{SEM}$, ( $n=6$ per group). ${ }^{*} P<0.05 \mathrm{vs}$. Sham; ${ }^{*} P<0.05$ vs. vehicle. 


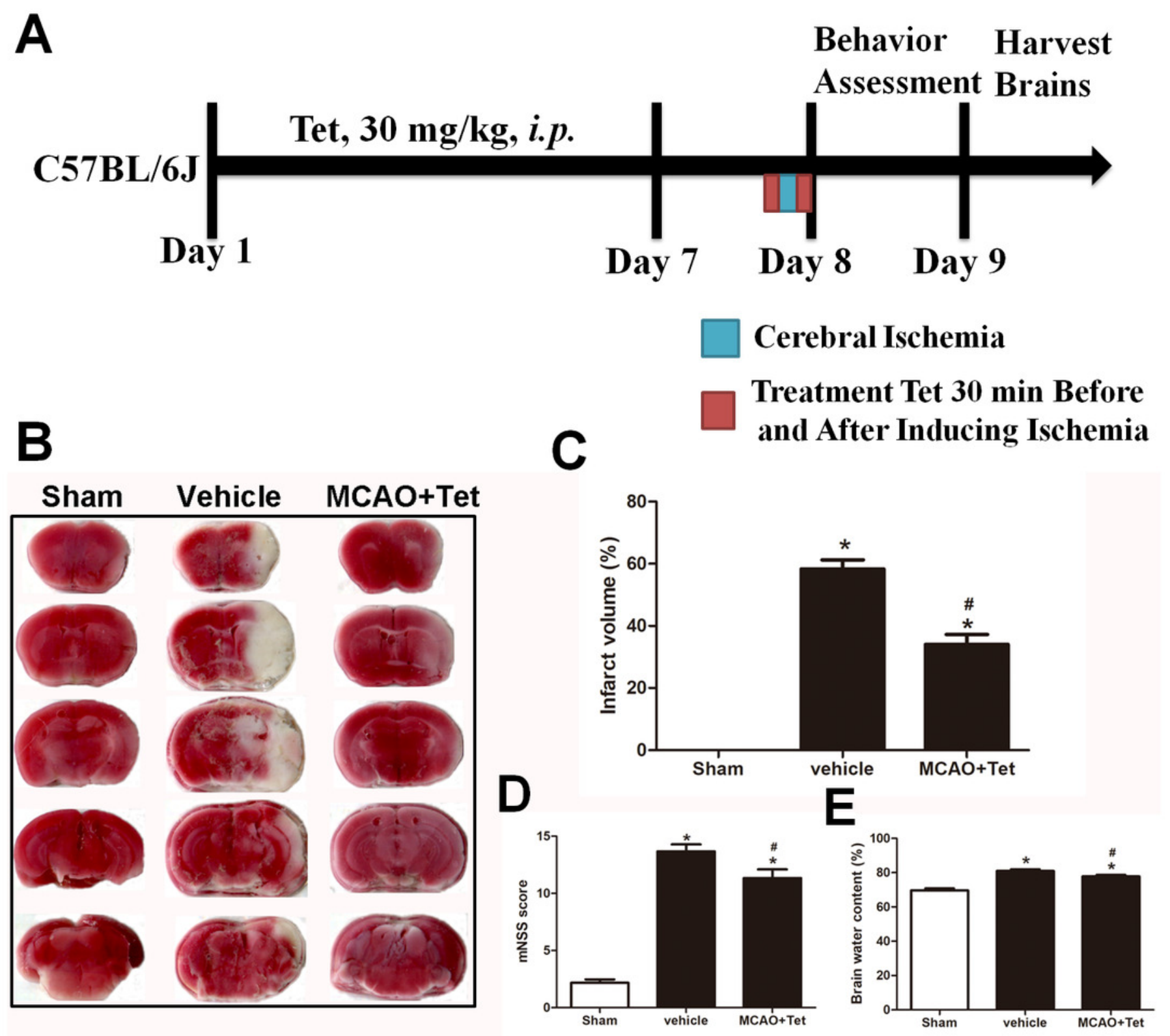




\section{Figure 2}

Tetrandrine inhibited the activation of the NLRP3 inflammasome in hippocampal microglia of ischemic brain.

Brain sections were stained with DAPI (blue), as well as Iba-1 (green) or NLRP3 (red) to monitor NLRP3 accumulation. (A-L) Representative immunofluorescent staining in the hippocampus of mice. Scale bar, $100 \mu \mathrm{m}$. Iba-1 positive cells in the hippocampus (M) and percentage of cells with Iba-1 localization of NLRP3 (N) were quantified. Tet, Tetrandrine. 

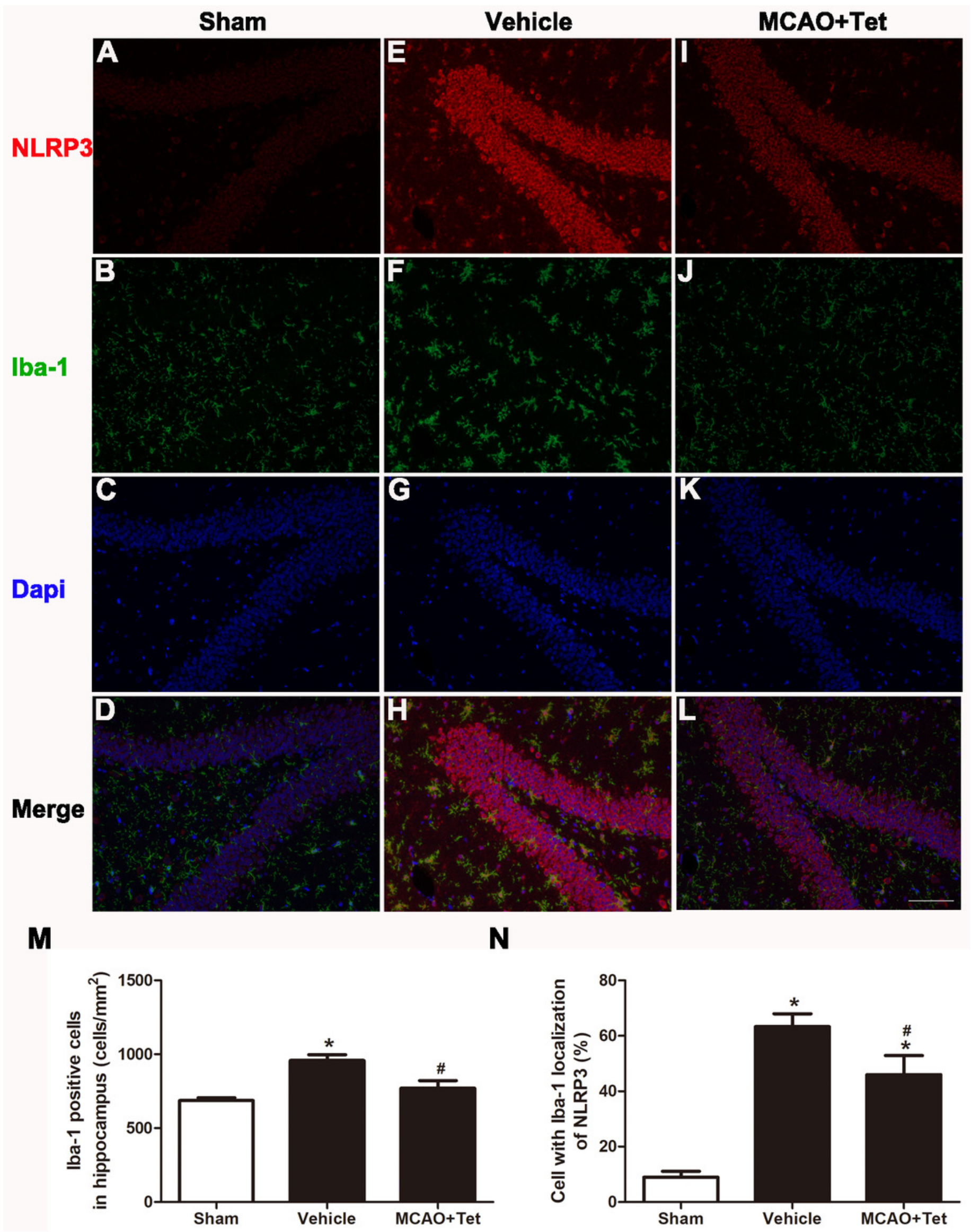


\section{Figure 3}

Tetrandrine inhibited NLRP3 -derived inflammation and upregulated Sirt-1 expression in ischemic brain in mice.

(A) Protein levels of NLRP3 and cleaved caspase-1 in brain tissues were measured by western blots and were normalized to GAPDH. The bar graphs show that Tet treatment clearly increased the expression of NLRP3 (B) and cleaved caspase-1 (C). N=3 per group. The levels of IL-1 $\beta$ (D) and IL-18 (E) were analyzed by ELISA. N=6 per group. The representative bands of western blots $(F)$ and quantification the Sirt-1 expression (G) were shown. Treatment with Tet before and after MCAO significantly increased Sirt-1 expression in brain tissues. $\mathrm{N}=3$ per group. Values are mean $\pm \mathrm{SEM},{ }^{*} P<0.05$ vs. Sham; ${ }^{\#} P<0.05$ vs. vehicle. Tet, Tetrandrine. 
A

\section{Sham Vehicle MCAO+Tet}

NLRP3

Cleaved caspase-1

GAPDH
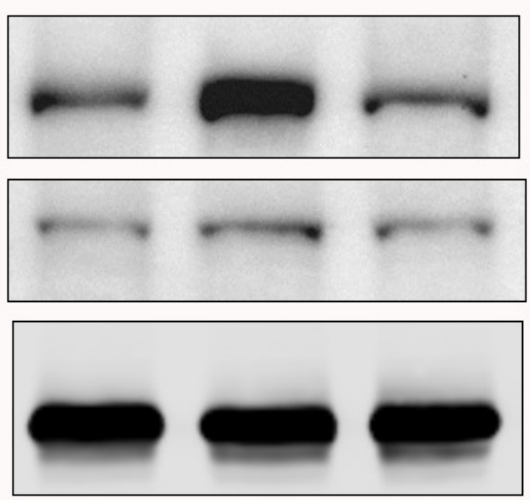

$106 \mathrm{KD}$

$45 \mathrm{KD}$

$36 \mathrm{KD}$

B
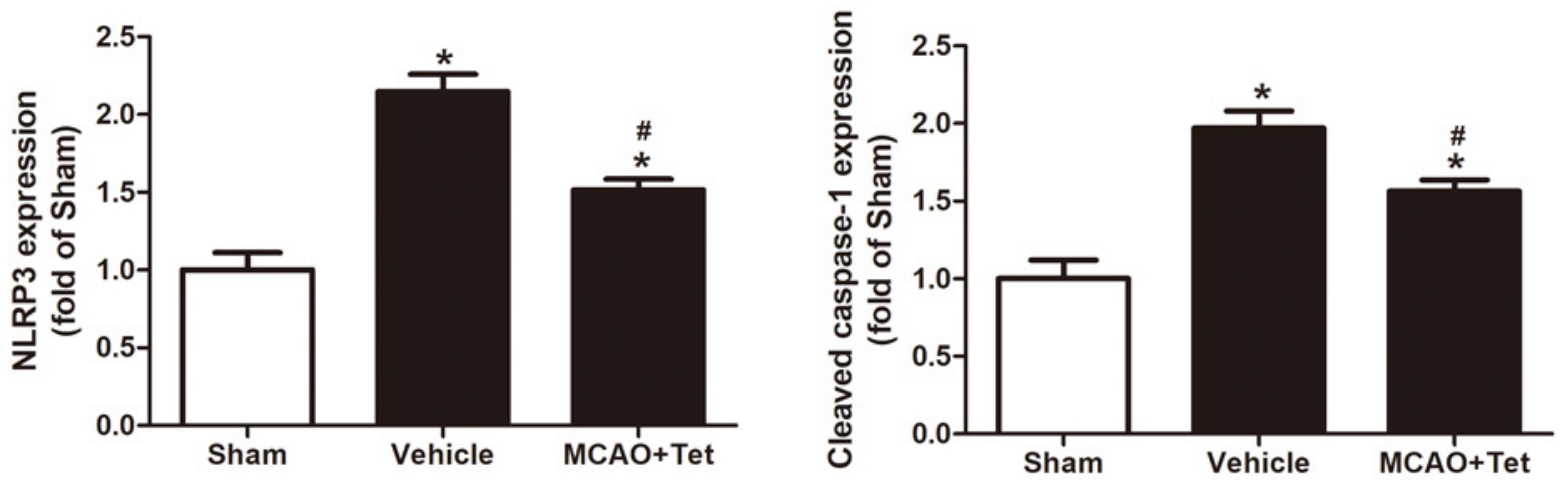

D
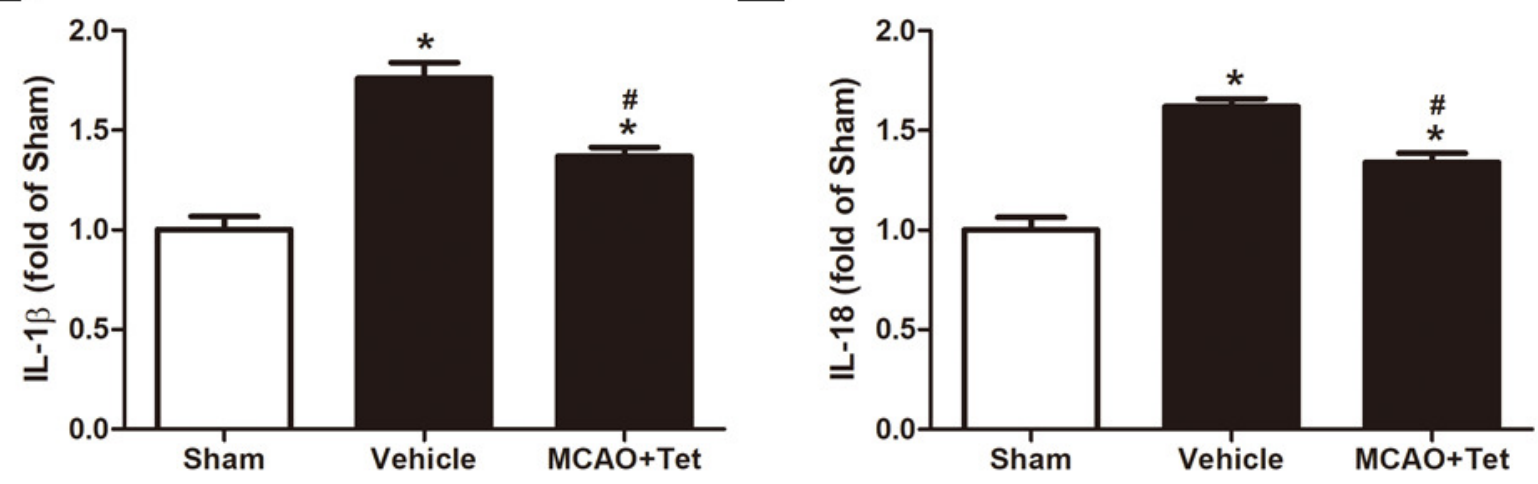

두

Sham Vehicle MCAO+Tet

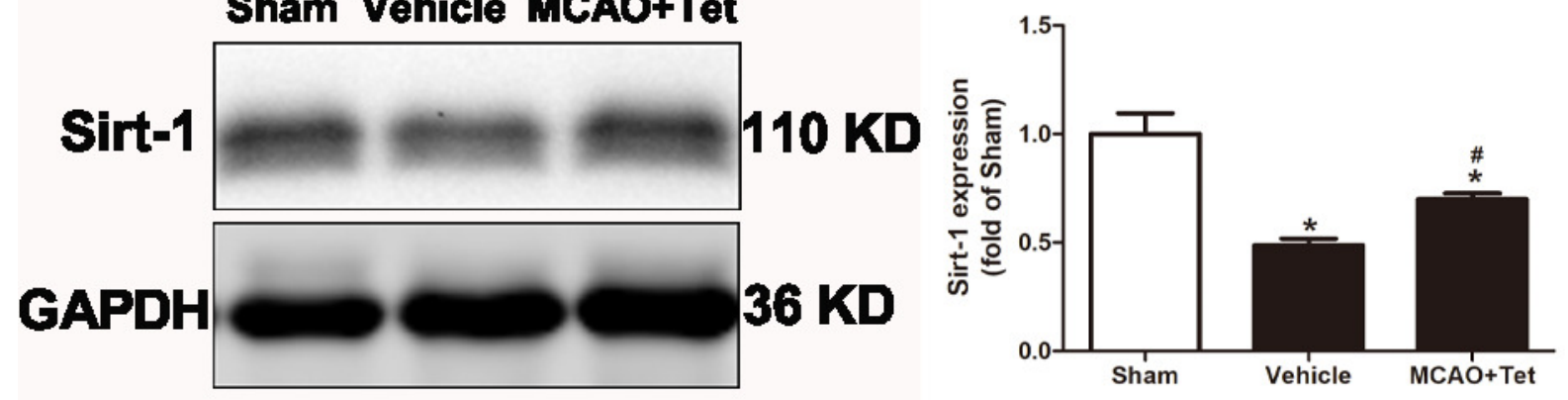




\section{Figure 4}

The effect of Sirt-1 siRNA pretreatment on infarct volumes and neurobehavioral outcomes in cerebral ischemia in mice.

An intracerebroventricular injection of Sirt-1 siRNA was administered to mice to inhibit cerebral Sirt-1 expression, and a scrambled siRNA was injected as a control. (A, B) TTCstained brain sections showed no significantly different atrophy between Sirt-1 siRNA group and Tet + Sirt- 1 siRNA group. $N=6$ per group, ${ }^{*} P<0.05$ vs. Tet. (C) Neurological severity scores were detected after cerebral ischemia. $N=6$ per group. ${ }^{*} P<0.05$ vs. Tet. (D) Representative western blots showed the protein level of Sirt-1 in mice and was normalized to GAPDH. Sirt-1 siRNA pretreatment significantly inhibited protein level of Sirt-1 in brain tissues. Quantification of the expression level is shown in (E). $N=3$ per group. ${ }^{*} P<0.05$. Values are mean \pm SEM. Tet, Tetrandrine. NS, no significance.

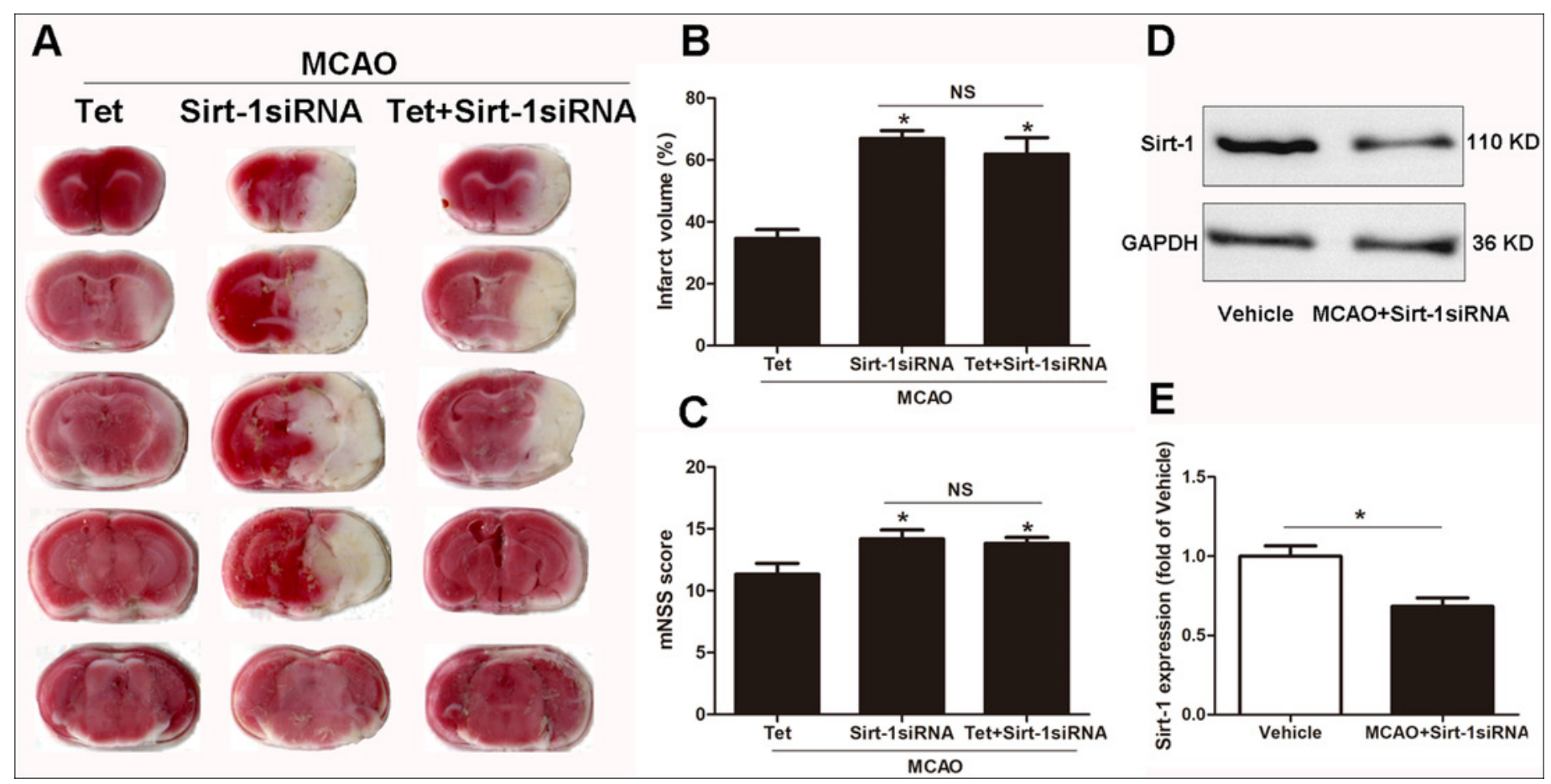




\section{Figure 5}

Sirt-1 siRNA obviously reverses the effects of Tetrandrine on expression of NLRP3, IL-1 $\beta$ and IL-18 in mice.

Tet and Sirt-1 siRNA pretreatment blocked the Tet-induced decrease NL RP3 expression in Ib a-1-positive cells in the hippocampus (A-C). Scale bar, $100 \mu \mathrm{m}$. Iba-1 positive cells in the hippocampus (D) and percentage of cells with Iba-1 localization of NLRP3 (E) were quantified. Sirt-1 siRNA also blocked the Tet-induced reduction of IL-1 $\beta$ (F) and IL-18 (G) levels. Values are mean \pm SEM, $\left(n=6\right.$ per group). ${ }^{*} P<0.05$ vs. Tet. Tet, Tetrandrine. NS, no significance. 
Tet

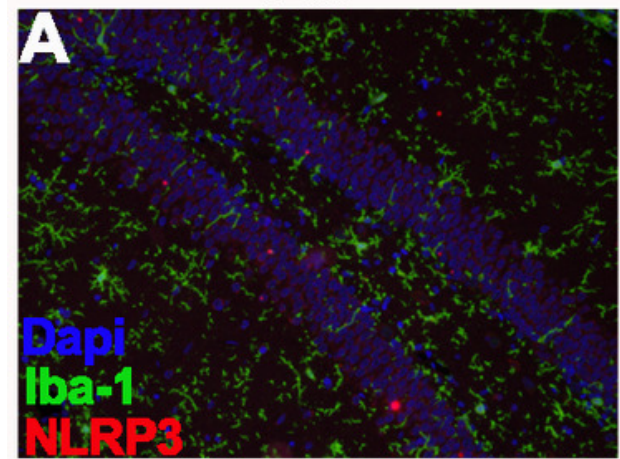

D

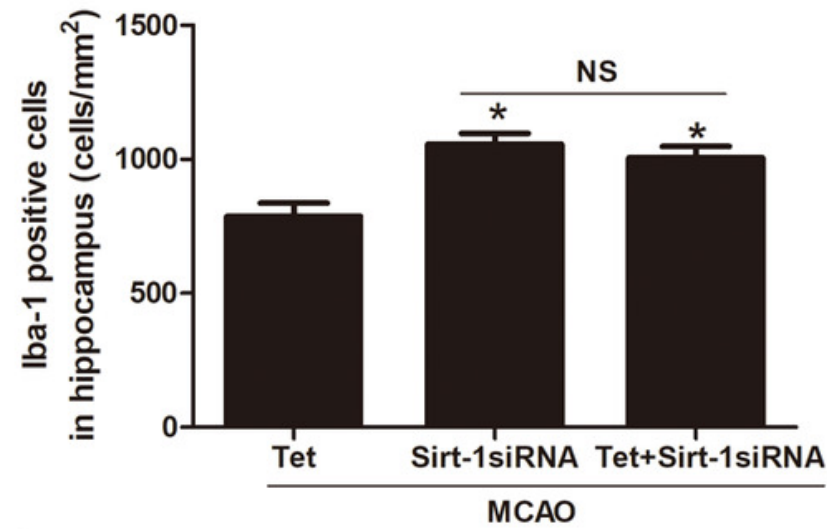

F

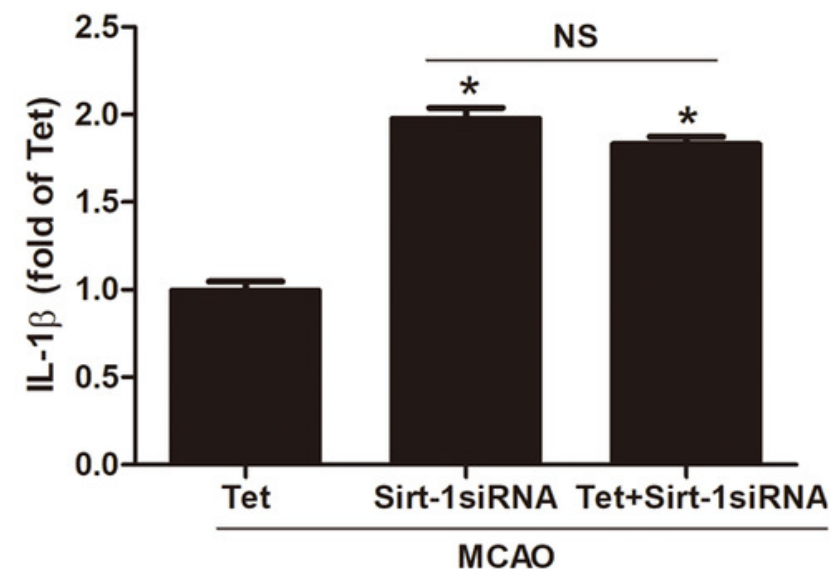

Sirt-1siRNA

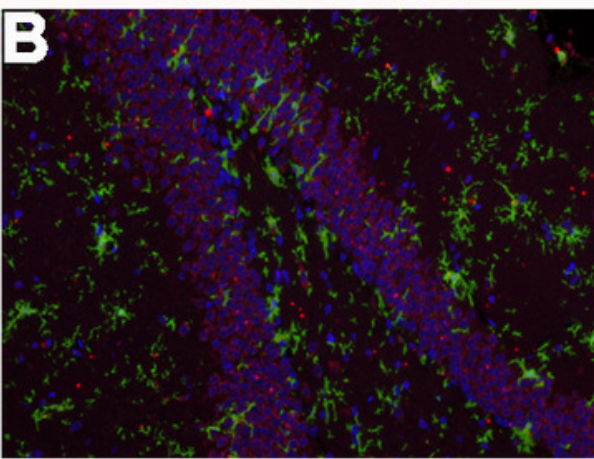

E

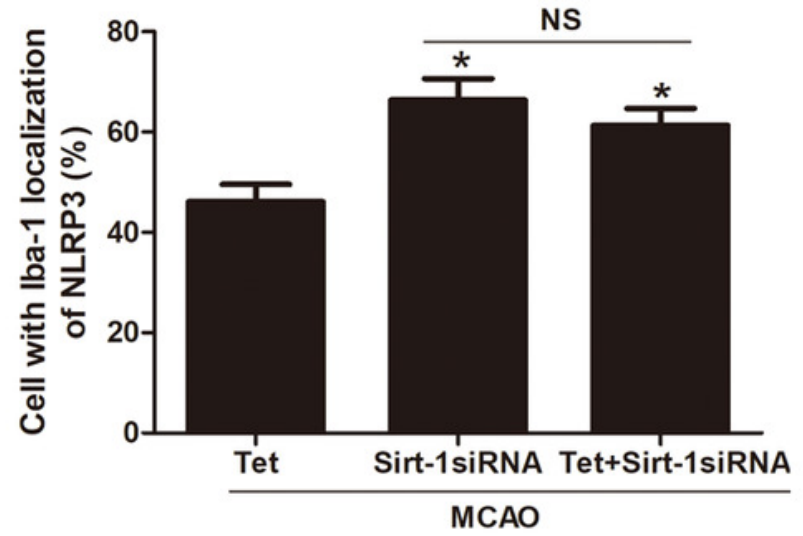

G

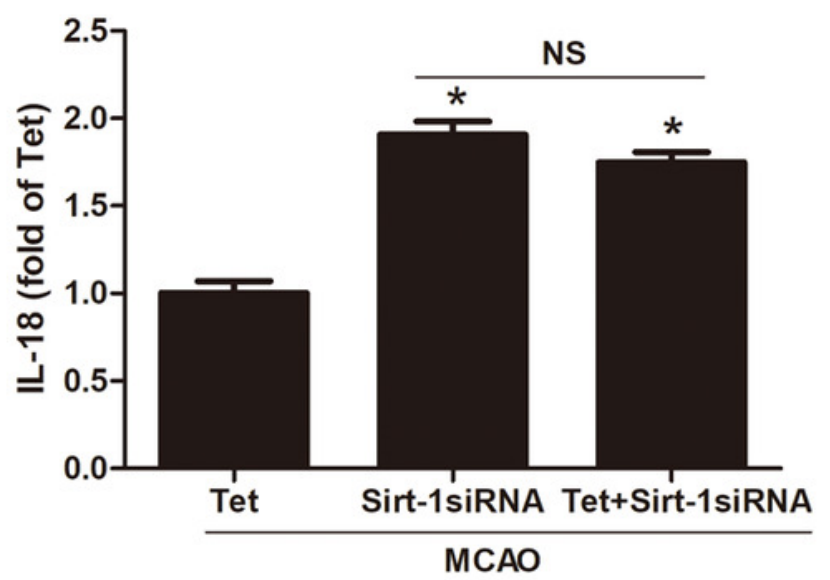

